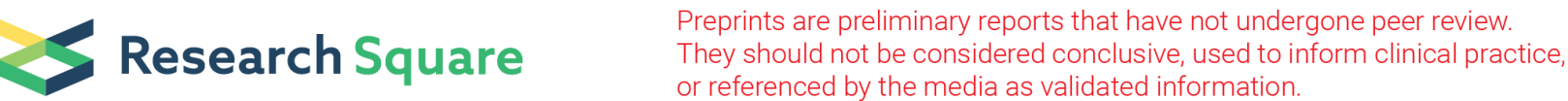

\section{Telerehabilitation program for older adults on a waiting list for physical therapy after hospital discharge: study protocol for a pragmatic randomized trial protocol}

\section{Pollyana Ruggio Tristao Borges}

UFMG: Universidade Federal de Minas Gerais https://orcid.org/0000-0002-6703-1536

Renan Resende ( $\square$ renan.aresende@gmail.com )

UFMG: Universidade Federal de Minas Gerais https://orcid.org/0000-0002-1609-3278

Jane Fonseca Dias

Universidade Federal de Minas Gerais

Marisa Cotta Mancini

Universidade Federal de Minas Gerais

Rosana Ferreira Sampaio

Universidade Federal de Minas Gerais

\section{Study protocol}

Keywords: Older adults, Deconditioning, Telerehabilitation, Physical Therapy

Posted Date: June 14th, 2021

DOI: https://doi.org/10.21203/rs.3.rs-397821/v1

License: (c) (1) This work is licensed under a Creative Commons Attribution 4.0 International License.

Read Full License

Version of Record: A version of this preprint was published at Trials on July 13th, 2021. See the published version at https://doi.org/10.1186/s13063-021-05387-2. 


\section{Abstract}

Background: Delays in starting physical therapy after hospital discharge worsen deconditioning in older adults. Intervening quickly can minimize the negative effects of deconditioning. Telerehabilitation is a strategy that increases access to rehabilitation, improves clinical outcomes, and reduces costs. This paper presents the protocol for a pragmatic clinical trial that aims to determine the effectiveness and cost-effectiveness of a multi-component intervention offered by telerehabilitation for discharged older adults awaiting physical therapy for any specific medical condition.

Methods: This is a pragmatic randomized controlled clinical trial with two groups: telerehabilitation and control. Participants $(n=230)$ will be recruited among individuals discharged from hospitals who are in the public healthcare system physical therapy waiting lists. The telerehabilitation group will receive a smartphone app with a personalized program (based on individual's functional ability) of resistance, balance, and daily activity training exercises. The intervention will be implemented at the individuals' homes. This group will be monitored weekly by phone and monthly through a face-to-face meeting until they start physical therapy. The control group will adhere to the public healthcare system usual flow and will be monitored weekly by telephone until they start physical therapy. The primary outcome will be physical function (Timed Up and Go and 30-second Chair Stand Test). The measurements will take place in baseline, start, and discharge of outpatient physical therapy. The economic evaluations will be performed from the perspective of society and the Brazilian public healthcare system.

Discussion: The study will produce evidence on the effectiveness and cost-effectiveness of multicomponent telerehabilitation intervention for discharged older adult patients awaiting physical therapy, providing input that can aid implementation of similar proposals in other patient groups.

Trial registration: Brazilian Clinical Trials Registry, RBR-9243v7. Registered on 24 August 2020.

\section{Background}

Deconditioning causes significant functional decline in older adults and is often associated with readmissions, institutionalization, and mortality. ${ }^{1,2,3}$ Deconditioning in older adults refers to a systemic physiological change after a period of inactivity and/or immobility. ${ }^{2,4,5,6}$ These changes may begin during hospitalization, as many older people are confined to bed. ${ }^{7,8}$ Studies demonstrate that deconditioning remains after discharge ${ }^{9,10}$ and the older adults who resume physical therapy immediately after leaving hospital show gains. ${ }^{11,12}$ The literature recommends that deconditioned older adults should receive moderate to high-intensity training focused on resistance exercises, walking, balance, and daily life activities. $^{2}$

Older people referred for rehabilitation after discharge may face long waiting lists to start treatment. The imbalance between rehabilitation supply and the growing demand for its service leads to increased waiting time for treatment. ${ }^{13}$ This is especially common in low and middle-income countries such as 
Brazil, ${ }^{14}$ whose public healthcare system serves approximately $70 \%$ of the population. ${ }^{15}$ Specifically in the city of Belo Horizonte, located in the southeastern Brazilian state of Minas Gerais, the delay for starting physical therapy treatment in the public healthcare system can take up to three months. Approximately 400 individuals of different ages are waiting for physical therapy; of these, almost $70 \%$ are hospital discharges with different types of medical conditions such as postoperative of lower or upper limbs, fractures, and COVID-19. Inactivity during this waiting period may aggravate deconditioning, especially in older adults.

Telerehabilitation may be a viable alternative to reduce the waiting time for physical therapy, ${ }^{16}$ minimizing the negative impacts of inactivity impacts of inactivity, such as falls, ${ }^{17}$ sedentary pattern, ${ }^{18}$ and loss of strength, mobility and resistance. ${ }^{19}$ Telerehabilitation provides rehabilitation through information and communication technologies such as videoconferencing, telephoning, and smartphone app. ${ }^{21,22,23}$ This rehabilitation modality offers early access to treatment, reduces costs (mostly with transportation), induces the patient to play a more active role in the treatment, and allows the treatment to be adapted to their routine. ${ }^{16,24,25,26,27}$ Evidence shows that telerehabilitation is safe, ${ }^{28,29}$ effective, and less costly when compared to face-to-face rehabilitation. ${ }^{24,26,27}$ In patients who underwent orthopedic surgery, telerehabilitation had positive effects on physical function, ${ }^{29,30}$ range of motion, ${ }^{31}$ function, and pain. ${ }^{32}$ In addition, the face-to-face and telerehabilitation modalities demonstrated equivalent results for hospital discharged patients. ${ }^{33,34,35,36,37,38}$ More recently, the social isolation resulting from the COVID-19 pandemic has shown that distance healthcare is essential to ensure treatment continuity, especially for older adults. ${ }^{39}$ Therefore, investing in programs that reduce costs without compromising the population's access is essential.

This paper presents the protocol for a pragmatic superiority clinical trial that aims to determine the effectiveness and cost-effectiveness of a personalized exercise program offered by telerehabilitation to minimize the deconditioning of older adults who are, for non-specific conditions, awaiting outpatient physical therapy.

\section{Methods}

\section{Study design}

This is a protocol for a pragmatic randomized controlled clinical trial. Pragmatic trials are used to evaluate the effectiveness of interventions in the actual clinical practice setting. This design maximizes the application and generalization of results by establishing an appropriate basis for decisionmaking. ${ }^{40,41}$ This study protocol has been reported in accordance with the Standard Protocol Items: Recommendations for Interventional Trials (SPIRIT) Statement. ${ }^{42}$ Additional file 1 details the SPIRIT checklist.

\section{Setting and Participants}


Participants will be recruited from the waiting list for outpatient physical therapy of the Municipal Health Secretariat of Belo Horizonte, Brazil. All individuals on this list aged $\geq 60$ years and referred to physical therapy after hospital discharge will be contacted by telephone for an initial survey to determine whether they fit the inclusion criteria with questions on mobility, cognition, and internet access. At an in-person meeting, the researchers will detail the study and clarify any questions. The participant will then be asked to sign the consent form and will undergo the initial evaluation. Figure 1 details the study planning.

The inclusion criteria are: 1 ) older adults age $\geq 60$ years; ${ }^{43}$ 2) being in a waiting list for outpatient physical therapy in the public healthcare system for any specific medical condition; 3 ) have recently been discharged from the hospital; 4) no impediment to unload weight in the lower limbs to perform the telerehabilitation program; 5) walking independently or with the aid of a device and being able to sit and stand up from a chair to perform physical function tests; 6 ) being in a stable clinical condition to avoid complications during the telerehabilitation program; 7) having a smartphone device with internet access (their own or a companion's) for the telerehabilitation intervention; 8) having a companion during the execution of the exercises at home to ensure the safety of the intervention.

The exclusion criteria are: 1) presence of clinical complications that make physical exercise impossible; 2) neurological disease, such as Parkinson or stroke; 3 ) a score in the Mini-Mental State Exam less than 13 for people with no schooling, 18 for individuals with low/moderate schooling, and 26 for people with a high level of schooling; ${ }^{44} 4$ ) not being able to understand instructions or complete the tests. Excluded individuals will receive a booklet on the importance of staying active while awaiting physical therapy.

\section{Randomization and allocation}

After the initial evaluation, participants will be randomly allocated to the following groups: telerehabilitation (TG) and control (CG). The allocation will be in random blocks sizing four and six, with an allocation rate of 1:1. The randomized sequence will be computer-generated before the start of the study by a researcher not involved in the study and placed in opaque, sealed, sequentially numbered envelopes. This sequence will remain concealed until the participant is allocated to the groups.

\section{Telerehabilitation intervention}

The TG will receive a multi-component intervention that includes resistance exercises targeting the main muscle groups of the lower and upper limbs, and balance exercises. There are strong recommendations for the use of this type of intervention, showing improvement in clinical outcomes in older adults, such as reducing falls and increasing muscle strength and mobility. 2,17,18,19,20,45 These exercises are easy to perform, do not require special equipment or accessories, and were designed for execution without professional supervision. In addition, the participant will choose an activity from their daily routine that they have difficulty in performing and hope to improve in the short term. The participants' choosing an activity is a strategy aimed to stimulate engagement in the intervention and increase of their independence. 
The exercise program will be carried out three to five times a week with high intensity, as suggested by Falvey et al. (2015). ${ }^{2}$ The volume will have three series; the number of repetitions will be customized based on Borg's Modified Perceived Exertion Scale, ${ }^{46}$ with effort level ranging from five to seven. The exercises' difficulty levels and their sublevels of progression are shown in Table 1 and Additional file 2. Each participant may carry out the entire exercise program once a day or intermittently, two or three times a day.

The exercises will be available in a smartphone app developed for this study. This technology is widely used, being the main form of Internet access. ${ }^{47}$ The app will be installed on either the participant's or the companion's smartphone. After each exercise, the participants will report their level of pain and difficulty on the app, on a scale of 0 to 5 . The exercise program may also be accessed via a computer if the participant prefers this device. After the initial evaluation, participants and their companion will be trained in how to use the app and perform the exercises. Information on deconditioning and the benefits of the proposed exercises will also be provided.

Two previously trained physical therapists who have a master's degree will be responsible for monitoring the intervention. The proposed exercises and activities will be performed at home, in the presence of a companion to increase the safety of the exercises and facilitate the management of the app. Participants will be monitored by telephone and in-person meetings. The phone calls will take place weekly to monitor the exercises execution, pain, and adverse events. The progression of the exercises' difficulty through the protocol's sublevels will be based on this information, as well as those reported daily in the app. The faceto-face meetings will occur monthly (between 24-30 days) at the participant's rehabilitation center. In these meetings, the professionals will evaluate the execution of the exercises and adjust the difficulty level according to the participant's performance. The intervention will cease when the participant is contacted to begin outpatient physical therapy. Therefore, the participation period in the trial will depend on the waiting time of each participant.

\section{Control group}

CG participants will follow the usual flow of the waiting list of patients awaiting outpatient physical therapy in the public healthcare system and will not receive any guidance or exercise, as recommended in pragmatic studies. They will be monitored weekly by telephone call to check the onset of outpatient physical therapy, and adverse events. Like the TG, the period each CG participant will spend in the trial will depend on the time awaiting outpatient physical therapy. Participants in both the TG and CG will not be prohibited from receiving others interventions such as exercises on their own, physical activity (eg. walking), medications, or physical therapy elsewhere while on the waiting list, but this information will be recorded.

\section{Data collection}

Figure 2 shows the schedule of enrollment, interventions, and assessments (according to the SPIRIT). Outcomes will be assessed by in-person meetings at baseline (t0), at the end of the telerehabillitation 
protocol (t1), and after discharge from outpatient physical therapy or within six months of outpatient treatment (t2). All evaluations will be performed by a trained evaluator blinded to group allocation. All instruments show adequate validity and reliability for the older adults. $48,49,50,51,52$

In the baseline (t0), besides the outcomes, socio-demographic and clinical data will be collected, as well as data on the companion, and information on the use of communication technologies.

In the second evaluation (t1), satisfaction with the intervention and the TG app will also be evaluated. To minimize sample loss, the second evaluation will occur before the start of outpatient physical therapy or up to one week after the start of physical therapy treatment. The start of the outpatient physical therapy will be accompanied by weekly phone calls to both groups.

The final evaluation (t2) will take place on the last day of outpatient physical therapy or up to one week after discharge. The evaluation will consist of the collection of outcomes and information about outpatient physical therapy. Reasons for program discontinuity and follow-up losses will be recorded throughout the study.

\section{Outcomes}

\section{Primary outcome}

The primary outcome will be physical function measured by the Timed Up and Go (TUG) and the 30second Chair Stand Test (30CST). These tests were chosen because they are simple, fast, widely used, and do not require special equipment.

The TUG was developed to measure the functional mobility of older people. ${ }^{53}$ The participant gets up from a chair and walks three meters alone or with the aid of a walking device, at a comfortable and safe speed, and then return and sit down again in the same chair. ${ }^{53}$

The 30CST consists of sitting and standing, tasks often performed daily. ${ }^{54,55}$ This test was developed as a measure of lower limb strength for older adults. ${ }^{49}$ The participant sits in a chair with arms crossed in front of the torso and must repeatedly stand and sit for 30 seconds as fast as possible. ${ }^{56}$

\section{Secondary outcomes}

The Physical Functioning Scale of the Brazilian version of the Medical Outcomes Short-Form Health Survey (SF-36) will be used to measure physical health. ${ }^{57}$ The Physical Functioning Scale presents ten questions; each one is scored from 1 to 3 according to the perceived limitation. ${ }^{58}$ The final score of the scale is calculated according to the orientation of the SF-36 authors ranges from 0-100, with lower scores indicating higher limitations. ${ }^{58}$

Pain will be measured by the visual analog scale with numerical (0-10) and color gradations. ${ }^{59}$ 
Health-related quality of life will be measured by the Brazilian version of EuroQol-5D (EQ-5D-3L). This instrument evaluates the patient's current health status in five dimensions (mobility, self-care, usual activity, pain/discomfort, and anxiety/depression). EQ-5D-3L also includes a visual analog scale to record overall health status. ${ }^{60}$ The results of this instrument are widely used in economic evaluations. ${ }^{61}$

Performance perception and satisfaction will be measured by the Canadian Occupational Performance Measure (COPM). ${ }^{62}$ In this study, only the self-care area will be used. The participant lists the self-care activities they have difficulty performing, chooses the one they consider the most important, and ranks their performance and performance satisfaction from 0 to 10 . Higher scores indicate better performance and performance satisfaction.

\section{Economic evaluation}

The economic evaluation will be carried out from the perspective of society and the Brazilian public healthcare system during the study period. The costs will be reported based on values updated to the year of data collection. In this phase, all information will follow the recommendations of the Brazilian Ministry of Health's Methodological Guidelines for Economic Evaluation. ${ }^{63}$ Cost measures will be obtained by estimates of health service utilization (public and private) due to the health condition that caused the referral to outpatient physical therapy and/or generated adverse events. The app development costs will be excluded.

Costs to the public healthcare system will be measured based on the use of health services and procedures reported by participants by weekly Google form platform and these data will only be assessed by researchers blinded to group allocation. Costs for hospital care, elective consultations, outpatient or home physical therapy, and medication will be included. These costs will be valued using standardized cost tables of the Brazilian public healthcare system.

Costs to society will include direct costs to participants and caregivers and loss of productivity. The calculation of direct expenses, such as medication, purchase of equipment, transportation, hiring caregivers, and health services will be based on surveys answered by participants. The cost of transportation will be evaluated by the distance between the participant's home and the physical therapy site, adjusted by the type of transportation used and the price of gasoline. Information on loss of productivity will be obtained for participants and non-contracted caregivers (e.g. family members) through surveys administered to participants who will indicate whether they have paid work, income from this work, and lost workdays due to the health condition.

The cost-consequence approach will be used to compare the results obtained by the participants in the primary and secondary outcomes with the costs for the two perspectives of analysis (society and public healthcare system). In the cost-utility analysis, the costs for the public healthcare system will be compared with the quality-adjusted life years (QALY) gained from the intervention, obtained by applying the EQ-5D-3L. Sensitivity analyses will be conducted to explore any degree of uncertainty in the estimates, such as sampling, time horizon, discount rate, and imputation of missing data. 


\section{Other outcomes}

Adherence will be monitored through the records computed by the app and confirmed during the weekly calls to the TG. Participants who perform all the proposed exercises at least three times a week will be considered as adherents.

Adverse events will be monitored weekly during phone calls and will be reported in the study results. Adverse events are those harmful or unfavorable results that occur during or after exercise, ${ }^{64}$ such as nausea, headache, falling, and incapacitating pain. Events not related to the intervention will also be monitored. Participants who report a serious adverse event will be instructed to seek emergency medical services. The intervention will be interrupted if the adverse event makes it impossible for the participant to perform physical exercises.

\section{Sample size}

The sample size was based on the TUG primary outcome. An effect size of $d=0.4$, previously obtained by Brovold et al. (2012), ${ }^{65}$ power of $80 \%$, alpha of 0.05 , and abandonment rate of $15 \%$ were considered to estimate sample size using the $\mathrm{G}^{\star}$ Power 3.1 software. This resulted in a minimum sample size of 230 participants, 115 per group.

\section{Data analysis plan}

The outcome variables and the participants' characteristics will be analyzed with descriptive statistics. The Kolmogorov-Smirnov test will be used to test the normality of the data. Comparison of TG and CG baselines will be conducted with parametric or non-parametric test.

Outcome group comparisons will be analyzed with the nonlinear mixed effect model, ${ }^{66}$ given that deconditioning follows a nonlinear path over time and the period individuals remain in the study is not homogeneous. Since the intervention time will be different among the participants, time will be treated as a random factor.

All data will be analyzed for intention-to-treat; that is, all random participants will be included in the analysis regardless of adherence to the protocol. The data will be analyzed by the R software ( $R$ Foundation for Statistical Computing, Vienna, Austria) with a significance level of $5 \%$.

\section{Discussion}

\section{Potential impact and significance}

Our hypothesis is that the multi-component program offered by telerehabilitation will minimize the deconditioning of hospital discharged older adult patients waiting for physical therapy and will reduce costs. Older adults who were hospitalized due to different health conditions and who experienced functional decline have up to five times greater likelihood of dying and 10 to 20 times greater chance of 
being readmitted after hospital discharge. ${ }^{67,68,69}$ In addition, studies show that approximately $30 \%$ of these older individuals have functional losses at the moment of hospital discharge ${ }^{68,69}$ and that roughly the same proportion maintain these losses 30 days after discharge. ${ }^{69}$ The type of intervention most recommended for this population is a multicomponent program. ${ }^{2,45}$ Thus, the faster these older individuals start physical therapy and remove themselves from inactivity, the less negative will be the impacts and deconditioning advancement. Costs for patients and the public healthcare system may be reduced. Telerehabilitation reduces the cost of transportation. ${ }^{24,25,26}$ Once deconditioning is associated with readmissions ${ }^{1,2,3}$ and hospitalization generates cost to the healthcare system, the exercise program may reduce hospital readmissions.

In Brazil, telerehabilitation is still not a common practice, with a higher concentration of investments aimed towards telehealth. In low and middle-income countries, studies using remote rehabilitation are incipient. Due to the COVID-19 pandemic, this rehabilitation modality has been gaining prominence as an alternative to face-to-face treatment. ${ }^{70}$ The pandemic has impacted health services around the world, particularly non-urgent treatments. There is no estimate regarding the end of social isolation measures in many countries, and it is not possible to predict when healthcare services will return to normality. Waiting lists for outpatient services will possibly be even longer after the pandemic. As such, telerehabilitation is becoming increasingly essential to ensure treatment for the general population.

\section{Strengths and weakness}

This pragmatic study will be developed in the context of the public healthcare system, which favors-if our hypothesis is proven right-a simple and direct application in clinical practice. To reduce the risk of bias, the protocol will be prospectively recorded, the sample size will be representative of the population of interest, participants will be randomly allocated to groups, the allocation process will be concealed, the evaluators will be blinded to the group allocation and data will be analyzed by intention-to-treat. Blinding participants and physical therapists to the intervention will not be possible due to the nature of the intervention.

\section{Contribution to physical therapy}

We expect the clinical trial will provide evidence on the effectiveness and cost-effectiveness of a multicomponent telerehabilitation program for hospital discharged older adult patients awaiting outpatient physical therapy in the Brazilian public healthcare system. In addition, the study is expected to trigger discussions and the implementation of similar interventions in different patient groups and locations.

\section{Trial status}

Protocol version 1, date: August 24, 2020. Recruitment will start on February 22, 2021, http://www.ensaiosclinicos.gov.br/rg/RBR-9243v7. Recruitment completion is expected by December, 2021. The results of this trial will be submitted to a peer-reviewed journal after sample size is complete. 


\section{List Of Abbreviations}

30CST: 30-second Chair Stand Test

CG: control group

COPM: Canadian Occupational Performance Measure

EQ-5D-3L: EuroQol-5D

QALY: quality-adjusted life years

SF-36: Medical Outcomes Short-Form Health Survey

SPIRIT: Standard Protocol Items: Recommendations for Interventional Trials

TG: telerehabilitation group

TUG: Timed Up and Go

\section{Declarations}

\section{Ethics approval and consent to participate}

All participants will be informed about the procedures and will sign the informed consent form obtained by a member of the research team. This study was approved by the Research Ethics Committee of the Universidade Federal de Minas Gerais, Brazil, and the Municipal Health Secretariat of Belo Horizonte (under registration CAAE: 30673820.8.0000.5149). Any amendments will be reported to the Ethics Committee, to the trial registry, and in future trial publications. Participant data will be safely and confidentially archived along with all trial documentation in numerical order for a period of 10 years after completion of the study. The participants will be treated by codes.

\section{Consent for publication}

Not applicable.

\section{Availability of data and materials}

The datasets used and/or analyzed during the current study will be available from the corresponding author on reasonable request.

\section{Competing interests}

The authors declare that they have no competing interest.

\section{Funding}


This study has received no external funding.

\section{Authors' contributions}

Concept/idea/research design: P.R.T. Borges, R.F. Sampaio, J.F. Dias, M.C. Mancini, R.A. Resende

Writing: P.R.T. Borges, J.F. Dias, R.F. Sampaio, M.C. Mancini, R.A. Resende

Data collection: P.R.T. Borges, J.F. Dias

Data analysis: P.R.T. Borges, F.F. Dias

Project management: R.F. Sampaio, R.A. Resende

Consultation (including review of manuscript before submitting): R.F. Sampaio, M.C. Mancini, R.A. Resende

\section{Acknowledgements}

The authors thank the Secretaria Municipal de Saúde de Belo Horizonte for permission to have access to the study settings, Leonardo Henrique da Silva Vales for the app development, and the Coordenação de Aperfeiçoamento de Pessoal Ensino Superior (CAPES) and Fundação de Amparo à Pesquisa de Minas Gerais (FAPEMIG) for providing doctoral and research scholarships.

\section{References}

1. Dharmarajan $K$, Hsieh AF, Lin Z, et al. Diagnoses and timing of 30-day readmissions after hospitalization for heart failure, acute myocardial infarction, or pneumonia. JAMA. 2013;309(4):355363. https://doi.org/10.1001/jama.2012.216476

2. Falvey JR, Mangione KK, Stevens-Lapsley JE. Rethinking Hospital-Associated Deconditioning: Proposed Paradigm Shift. Phys Ther. 2015;95(9):1307-1315. https://doi.org/10.2522/ptj.20140511

3. Brownlee SA, Blackwell RH, Blanco BA, et al. Impact of Post-Hospital Syndrome on Outcomes Following Elective, Ambulatory Surgery. Ann Surg. 2017;266(2):274-279. https://doi.org/10.1097/SLA.0000000000001965

4. Siebens $\mathrm{H}$, Aronow $\mathrm{H}$, Edwards D, Ghasemi Z. A randomized controlled trial of exercise to improve outcomes of acute hospitalization in older adults. J Am Geriatr Soc. 2000;48(12):1545-1552. https://doi.org/10.1111/j.1532-5415.2000.tb03862.x

5. Kortebein P. Rehabilitation for hospital-associated deconditioning. Am J Phys Med Rehabil. 2009;88(1):66-77. https://doi.org/10.1097/PHM.0b013e3181838f70

6. Krumholz HM. Post-hospital syndrome-an acquired, transient condition of generalized risk. N Engl J Med. 2013;368(2):100-102. https://doi.org/:10.1056/NEJMp1212324 
7. Brown CJ, Redden DT, Flood KL, Allman RM. The underrecognized epidemic of low mobility during hospitalization of older adults. J Am Geriatr Soc. 2009;57(9):1660-1665.

https://doi.org/10.1111/j.1532-5415.2009.02393.x

8. Covinsky KE, Pierluissi E, Johnston CB. Hospitalization-associated disability: "She was probably able to ambulate, but I'm not sure". JAMA. 2011;306(16):1782-1793.

https://doi.org/10.1001/jama.2011.1556

9. Luk JK, Chan CF. Rehabilitation outcomes of older patients at 6 months follow-up after discharged from a geriatric day hospital (GDH). Arch Gerontol Geriatr. 2011;52(3):327-330.

https://doi.org/10.1016/j.archger.2010.05.006

10. Huang HT, Chang CM, Liu LF, Lin HS, Chen $\mathrm{CH}$. Trajectories and predictors of functional decline of hospitalised older patients. J Clin Nurs. 2013;22(9-10):1322-1331.

https://doi.org/10.1111/jocn.12055

11. Martínez-Velilla N, Cadore EL, Casas-Herrero Á, Idoate-Saralegui F, Izquierdo M. Physical Activity and Early Rehabilitation in Hospitalized Elderly Medical Patients: Systematic Review of Randomized Clinical Trials. J Nutr Health Aging. 2016;20(7):738-751. https://doi.org/10.1007/s12603-016-0683-4

12. Kosse NM, Dutmer AL, Dasenbrock L, Bauer JM, Lamoth CJ. Effectiveness and feasibility of early physical rehabilitation programs for geriatric hospitalized patients: a systematic review. BMC Geriatr. 2013;13:107. https://doi.org/10.1186/1471-2318-13-107

13. World Health Organization (WHO). Meeting report. Rehabilitation 2030: A Call for Action. 2017. Available at: https://www.who.int/disabilities/care/Rehab2030MeetingReport_plain_text_version.pdf. Accessed 22 July 2020.

14. The World Bank (WDI). World Development Indicators (2019). Available at: https://databank.worldbank.org/reports.aspx?source=2\&country=BRA,UMC. Accessed 22 July 2020.

15. Ministério da Saúde (BR). Diretrizes estratégicas. Available at: https://bvsms.saude.gov.br/bvs/pacsaude/diretrizes.php. Accessed 22 July 2020.

16. Salisbury C, Montgomery AA, Hollinghurst S, et al. Effectiveness of PhysioDirect telephone assessment and advice services for patients with musculoskeletal problems: pragmatic randomised controlled trial. BMJ. 2013;346:f43. https://doi.org/10.1136/bmj.f43

17. Sherrington $C$, Fairhall NJ, Wallbank GK, et al. Exercise for preventing falls in older people living in the community. Cochrane Database Syst Rev.

2019;1(1):CD012424. https://doi.org/10.1002/14651858.CD012424.pub2

18. Brazo-Sayavera J, López-Torres O, Martos-Bermúdez Á, Rodriguez-Garcia L, González-Gross M, Guadalupe-Grau A. Effects of Power Training on Physical Activity, Sitting Time, Disability, and Quality of Life in Older Patients With Type 2 Diabetes During the COVID-19 Confinement. J Phys Act Health. 2021;21:1-9. https://doi.org/10.1123/jpah.2020-0489.

19. Morat M, Morat T, Zijlstra W, Donath L. Effects of multimodal agility-like exercise training compared to inactive controls and alternative training on physical performance in older adults: a systematic 
review and meta-analysis. Eur Rev Aging Phys Act. 2021;

25;18(1):4. https://doi.org/10.1186/s11556-021-00256-y

20. Gonçalves AK, Griebler EM, da Silva WA, Sant Helena DP, da Silva PC, Possamai VD, Martins VF. Does a Multicomponent Exercise Program Improve Physical Fitness in Older Adults? Findings From a 5Year Longitudinal Study. J Aging Phys Act. 2021;24:1-8. https://doi.org/10.1123/japa.2020-0070.

21. Seelman KD, Hartman LM. Telerehabilitation: policy issues and research tools. Int $J$ Telerehabil. 2009;1(1):47-58. https://doi.org/10.5195/ijt.2009.6013

22. Pramuka M, van Roosmalen L. Telerehabilitation technologies: accessibility and usability. Int J Telerehabil. 2009;1(1):85-98. https://doi.org/10.5195/ijt.2009.6016

23. Peretti A, Amenta F, Tayebati SK, Nittari G, Mahdi SS. Telerehabilitation: Review of the State-of-the-Art and Areas of Application. JMIR Rehabil Assist Technol. 2017;4(2):e7. https://doi.org/:10.2196/rehab.7511

24. Kairy D, Lehoux P, Vincent C, Visintin M. A systematic review of clinical outcomes, clinical process, healthcare utilization and costs associated with telerehabilitation. Disabil Rehabil. 2009;31(6):427447. https://doi.org/10.1080/09638280802062553

25. Eriksson L, Lindström B, Ekenberg L. Patients' experiences of telerehabilitation at home after shoulder joint replacement. J Telemed Telecare. 2011;17(1):25-30. https://doi.org/10.1258/jtt.2010.100317

26. Tousignant $M$, Moffet $H$, Nadeau $S$, et al. Cost analysis of in-home telerehabilitation for post-knee arthroplasty. J Med Internet Res. 2015;17(3):e83. https://doi.org/10.2196/jmir.3844

27. Fusco F, Turchetti G. Telerehabilitation after total knee replacement in Italy: cost-effectiveness and cost-utility analysis of a mixed telerehabilitation-standard rehabilitation programme compared with usual care. BMJ Open. 2016;6(5):e009964. https://doi.org/10.1136/bmjopen-2015-009964

28. Moffet $H$, Tousignant $M$, Nadeau S, et al. In-Home Telerehabilitation Compared with Face-to-Face Rehabilitation After Total Knee Arthroplasty: A Noninferiority Randomized Controlled Trial. J Bone Joint Surg Am. 2015;97(14):1129-1141. https://doi.org/10.2106/JBJS.N.01066

29. Wang X, Hunter DJ, Vesentini G, Pozzobon D, Ferreira ML. Technology-assisted rehabilitation following total knee or hip replacement for people with osteoarthritis: a systematic review and metaanalysis. BMC Musculoskelet Disord. 2019;20(1):506. https://doi.org/10.1186/s12891-019-2900-x

30. Agostini M, Moja L, Banzi R, et al. Telerehabilitation and recovery of motor function: a systematic review and meta-analysis. J Telemed Telecare. 2015;21(4):202-213.

https://doi.org/10.1177/1357633X15572201

31. Shukla H, Nair SR, Thakker D. Role of telerehabilitation in patients following total knee arthroplasty: Evidence from a systematic literature review and meta-analysis. J Telemed Telecare. 2017;23(2):339346. https://doi.org/0.1177/1357633X16628996

32. van Egmond MA, van der Schaaf $M$, Vredeveld $T$, et al. Effectiveness of physiotherapy with telerehabilitation in surgical patients: a systematic review and meta-analysis. Physiotherapy. 2018;104(3):277-298. https://doi.org/10.1016/j.physio.2018.04.004 
33. Dias JF, Oliveira VC, Borges PRT, et alEffectiveness of exercises by telerehabilitation on pain, physical function and quality of life in people with physical disabilities: a systematic review of randomised controlled trials with GRADE recommendationsBritish Journal of Sports Medicine Published Online First: 15 October 2020. https://doi.org/10 10.1136/bjsports-2019-101375

34. Eriksson L, Lindström B, Gard G, Lysholm J. Physiotherapy at a distance: a controlled study of rehabilitation at home after a shoulder joint operation. $J$ Telemed Telecare. 2009;15(5):215-220. https://doi.org/10.1258/jtt.2009.081003

35. Pain, $H$, Soopramanien A, Dallolio L, Prior R, Menarini M, Ventura $M$, et al. Outcomes from a randomized controlled trial of telerehabilitation for people with spinal cord injuries. $J$ Telemed Telecare. 2007;13(1), 46-48. https://doi.org/10.1258/135763307781645095

36. Russell TG, Buttrum P, Wootton R, Jull GA. Internet-based outpatient telerehabilitation for patients following total knee arthroplasty: a randomized controlled trial. J Bone Joint Surg Am. 2011;93(2):113-120. https://doi.org/10.2106/JBJS.I.01375

37. Tousignant $M$, Moffet $H$, Boissy $P$, Corriveau $H$, Cabana F, Marquis F. A randomized controlled trial of home telerehabilitation for post-knee arthroplasty. J Telemed Telecare. 2011;17(4):195-198. https://doi.org/10.1258/jtt.2010.100602

38. Bini SA, Mahajan J. Clinical outcomes of remote asynchronous telerehabilitation are equivalent to traditional therapy following total knee arthroplasty: A randomized control study. J Telemed Telecare. 2017;23(2):239-247. https://doi.org/10.1177/1357633X16634518

39. Pedersini P, Corbellini C, Villafañe JH. Italian Physical Therapists' Response to the Novel COVID-19 Emergency. Phys Ther. 2020;100(7):1049-1051. https://doi.org/10.1093/ptj/pzaa060

40. Zwarenstein M, Treweek S, Gagnier JJ, et al. Improving the reporting of pragmatic trials: an extension of the CONSORT statement. BMJ. 2008;337:a2390. https://doi.org/10.1136/bmj.a2390

41. Thorpe $K E$, Zwarenstein $M, O x m a n ~ A D$, et al. A pragmatic-explanatory continuum indicator summary (PRECIS): a tool to help trial designers. J Clin Epidemiol. 2009;62(5):464-475. https://doi.org/10.1016/j.jclinepi.2008.12.011

42. Chan AW, Tetzlaff JM, Altman DG, et al. SPIRIT 2013 statement: defining standard protocol items for clinical trials. Ann Intern Med. 2013;158(3):200-207. https://doi:10.7326/0003-4819-158-3201302050-00583

43. World Health Organization (WHO). World report on ageing and health. 2015. Available at: https://apps.who.int/iris/handle/10665/186463. Accessed 19 May 2021.

44. Bertolucci PHF, Brucki SMD, Campacci SR, Juliano Y. O Mini-Exame do Estado Mental em uma população geral: impacto da escolaridade. Arq. Neuro-Psiquiatr.1994;52(1):01-

07. https://doi.org/10.1590/S0004-282X1994000100001

45. World Health Organization. WHO guidelines on physical activity and sedentary behaviour: at a glance: 2020. World Health Organization. Available at: https://apps.who.int/iris/handle/10665/337001. Accessed 10 Mai 2021.

46. Borg GA. Psychophysical bases of perceived exertion. Med Sci Sports Exerc. 1982;14(5):377-381. 
47. Instituto Brasileiro de Geografia e Estatística (IBGE). Acesso à internet e à televisão e posse de telefone móvel celular para uso pessoal: 2016. Pesquisa Nacional por Amostra de Domicílios (PNAD). Rio de Janeiro; 2018. Available at: https://biblioteca.ibge.gov.br/visualizacao/livros/liv101543.pdf. Accessed 22 July 2020.

48. Rydwik E, Bergland A, Forsén L, Frändin K. Psychometric Properties of Timed Up and Go in Elderly People: A Systematic Review.Physical \& Occupational Therapy In Geriatrics. 2011, 29:2, 102-125. https://doi.org/ 10.3109/02703181.2011.564725

49. Jones CJ, Rikli RE, Beam WC. A 30-s chair-stand test as a measure of lower body strength in community-residing older adults. Res Q Exerc Sport. 1999;70(2):113-119. https://doi.org/10.1080/02701367.1999.10608028

50. Lyons RA, Perry HM, Littlepage BN. Evidence for the validity of the Short-form 36 Questionnaire (SF36) in an elderly population. Age Ageing. 1994;23(3):182-184. https://doi.org/10.1093/ageing/23.3.182

51. Brazier JE, Walters SJ, Nicholl JP, Kohler B. Using the SF-36 and Euroqol on an elderly population. Qual Life Res. 1996;5(2):195-204. https://doi.org/10.1007/BF00434741

52. Tuntland $\mathrm{H}$, Kjeken I, Langeland $\mathrm{E}$, et al. Predictors of outcomes following reablement in communitydwelling older adults. Clin Interv Aging. 2016;12:55-63. https://doi.org/10.2147/CIA.S125762

53. Podsiadlo D, Richardson S. The timed "Up \& Go": a test of basic functional mobility for frail elderly persons. J Am Geriatr Soc. 1991;39(2):142-148. https://doi.org/10.1111/j.1532-5415.1991.tb01616.x

54. Riley PO, Schenkman ML, Mann RW, Hodge WA. Mechanics of a constrained chair-rise. J Biomech. 1991;24(1):77-85. https://doi.org/10.1016/0021-9290(91)90328-k

55. Dall PM, Kerr A. Frequency of the sit to stand task: An observational study of free-living adults. App/ Ergon. 2010;41(1):58-61. https://doi.org/10.1016/j.apergo.2009.04.005

56. Bohannon RW. Sit-to-stand test for measuring performance of lower extremity muscles. Percept Mot Skills. 1995;80(1):163-166. https://doi.org/10.2466/pms.1995.80.1.163

57. Ciconelli RM, Ferraz MB, Santos, W, Meinao IM, Quaresma MR. Brazilian-Portuguese version of the SF-36 questionnaire: A reliable and valid quality of life outcome measure. Arthritis And Rheumatism. 1997;40(9):489-489, 1997.

58. Ware JE, Snow KK, Kosinski M, Gandek B. SF-36 health survey: manual and interpretation guide. New England Medical Center Hospital. Health Institute. Boston: Health Institute, New England Medical Center; 1993.

59. Wewers ME, Lowe NK. A critical review of visual analogue scales in the measurement of clinical phenomena. Res Nurs Health. 1990;13(4):227-236. https://doi.org/10.1002/nur.4770130405

60. EuroQol Reserch Foundation. EQ-5D-3L User Guide, 2018. Available at: https://euroqol.org/publications/user-guides. Accessed July 22, 2020.

61. Santos M, Cintra MA, Monteiro AL, et al. Brazilian Valuation of EQ-5D-3L Health States: Results from a Saturation Study. Med Decis Making. 2016;36(2):253-263. https://doi.org/10.1177/0272989X15613521 
62. Law M, Baptiste S, McColl M, Opzoomer A, Polatajko H, Pollock N. The Canadian occupational performance measure: an outcome measure for occupational therapy. Can J Occup Ther. 1990;57(2):82-87. https://doi.org/10.1177/000841749005700207

63. Brasil. Secretaria de Ciência, Tecnologia e Insumos Estratégicos. Departamento de Ciência e Tecnologia. Diretrizes metodológicas: Diretriz de Avaliação Econômica.2a ed. Brasília: Ministério da Saúde; 2014.

64. Higgins JPT, Thomas J, Chandler J; Cumpston M, Li T, Page MJ et al. Cochrane Handbook for Systematic Reviews of Interventions version 6.1 (updated September 2020). Cochrane. 2020. Available at: www.training.cochrane.org/handbook. Accessed 19 Oct 2020.

65. Brovold T, Skelton DA, Bergland A. The efficacy of counseling and progressive resistance homeexercises on adherence, health-related quality of life and function after discharge from a geriatric day-hospital. Arch Gerontol Geriatr. 2012;55(2):453-459. https://doi.org/10.1016/j.archger.2012.01.015

66. Pinheiro JC, Bates DM. Mixed-effects models in S and S-PLUS. New York, NY: Springer; 2000.

67. Bellelli G, Magnifico F, Trabucchi M. Outcomes at 12 months in a population of elderly patients discharged from a rehabilitation unit. J Am Med Dir Assoc. 2008;9(1):55-64. https://doi.org/10.1016/j.jamda.2007.09.009

68. Covinsky KE, Palmer RM, Fortinsky RH, et al. Loss of independence in activities of daily living in older adults hospitalized with medical illnesses: increased vulnerability with age. J Am Geriatr Soc. 2003;51(4):451-458. https://doi.org/10.1046/j.1532-5415.2003.51152.x

69. Carvalho TC, Valle AP, Jacinto AF, Mayoral VFS, Boas PJFV. Impact of hospitalization on the functional capacity of the elderly: A cohort study. Rev. bras. geriatr. gerontol. 2018;21(2):134-142. https://doi.org/10.1590/1981-22562018021.17014

70. Turolla A, Rossettini G, Viceconti A, Palese A, Geri T. Musculoskeletal Physical Therapy During the COVID-19 Pandemic: Is Telerehabilitation the Answer?. Phys Ther. 2020;100(8):1260-1264. https://doi.org/10.1093/ptj/pzaa093

\section{Table}

Due to technical limitations the Table is available as a download in the Supplementary Files.

\section{Figures}




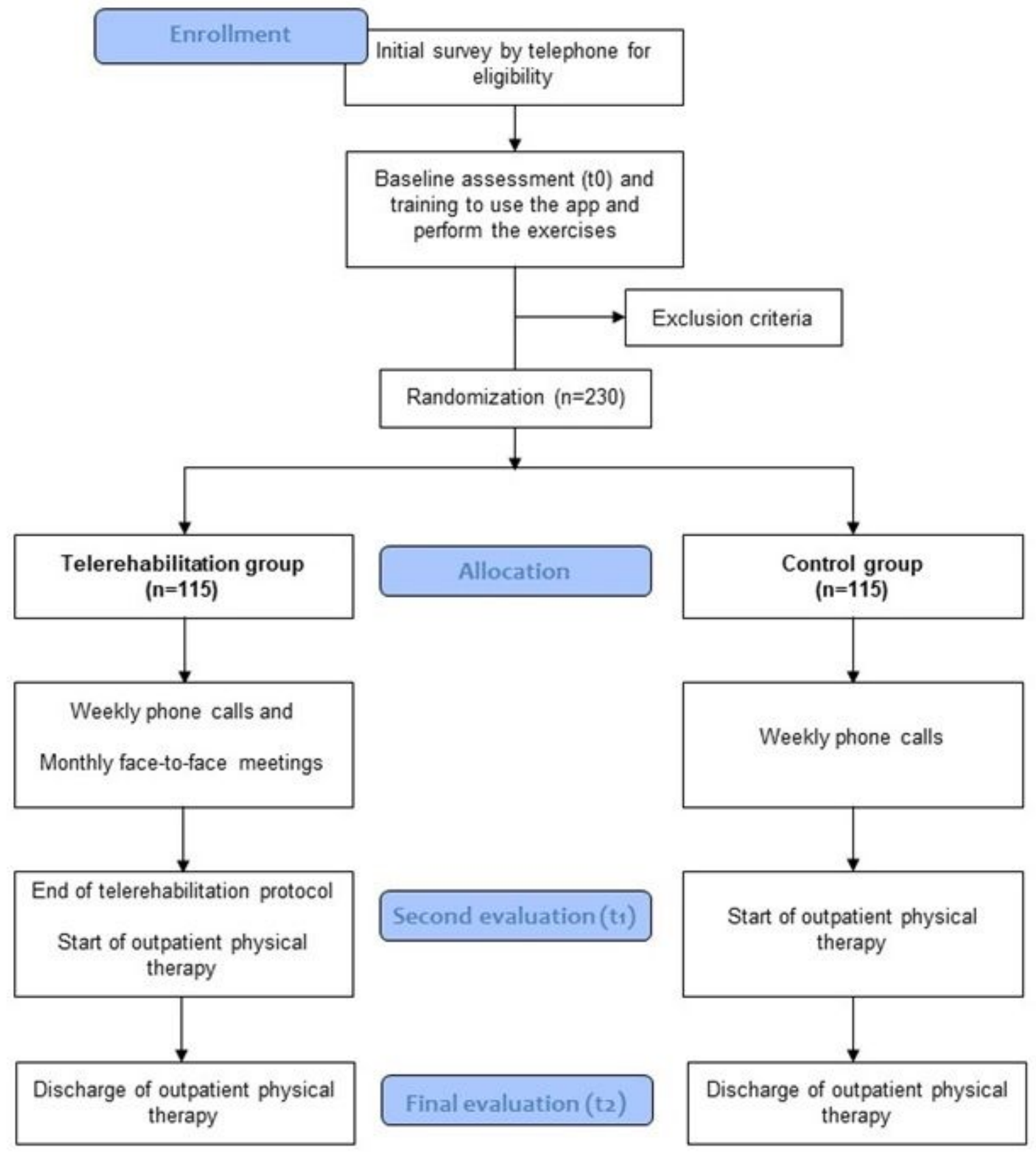

Figure 1

Flow diagram 


\begin{tabular}{|c|c|c|c|c|}
\hline & \multicolumn{4}{|c|}{ STUDY PERIOD } \\
\hline & Enrolment & Allocation & Post-allocation & Close-out \\
\hline TIMEPOINT & $t_{\text {p pousentioes }}$ & 0 & $t_{4}$ & $t_{2}$ \\
\hline \multicolumn{5}{|l|}{ ENROLMENT: } \\
\hline \multirow[b]{2}{*}{ Informed consent } & $\mathrm{x}$ & & & \\
\hline & $\mathrm{x}$ & & & \\
\hline Allocation & & $\mathrm{x}$ & & \\
\hline \multicolumn{5}{|l|}{ INTERVENTIONS: } \\
\hline \multicolumn{5}{|l|}{ Te/erehabilitation group } \\
\hline \multicolumn{5}{|l|}{ Control group } \\
\hline \multicolumn{5}{|l|}{ ASSESSMENTS: } \\
\hline Baseline variables & $\mathrm{x}$ & & & \\
\hline Primary outcomes & $\mathrm{x}$ & & & \\
\hline $30^{\prime}$ chair stand test & $\mathrm{x}$ & & & \\
\hline $\begin{array}{l}\text { Secondary outcomes } \\
\text { Pysical Functioning Scale } \\
\text { (SF-36) }\end{array}$ & $\mathrm{x}$ & & & \\
\hline Pain & $x$ & & & \\
\hline EuroQOL-SD & $x$ & & & \\
\hline $\begin{array}{l}\text { Canadian Occupational } \\
\text { Performance Measure }\end{array}$ & $\mathrm{x}$ & & & \\
\hline
\end{tabular}

\section{Figure 2}

Schedule of enrolment, interventions, and assessments (according to SPIRIT)

\section{Supplementary Files}

This is a list of supplementary files associated with this preprint. Click to download. 
- Additionalfile1SPIRITchecklist.doc

- Additionalfile2Exerciseprotocol.mp4

- CONSORTExtensionforPragmaticTrialsChecklist.docx

- Consentform.docx

- Datacollectionform.docx 